\title{
IMPLICACIONES DE LAS TAREAS ESCOLARES EN EL RENDIMIENTO ACADÉMICO EN LOS ESTUDIANTES DEL SÉPTIMO AÑO DE EDUCACIÓN GENERAL BÁSICA
}

\author{
AUTORES: Marjorie Narcisa Cevallos Rosero ${ }^{1}$ \\ Tania Estefanía Aguirre Espinosa² \\ Edgar Efraín Obaco Soto 3 \\ Cristopher David Herrera Navas ${ }^{4}$
}

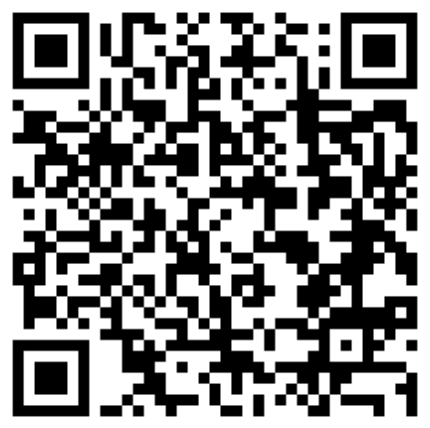

\section{DIRECCIÓN PARA CORRESPONDENCIA:mncevallosr@pucesd.edu.ec}

Fecha de recepción: 15/01/2020

Fecha de aceptación: 23/03/2020

\section{RESUMEN}

El propósito del presente estudio fue analizar el impacto de las tareas escolares en el rendimiento académico en los estudiantes de séptimo año de Educación General Básica de la Unidad Educativa Liceo de las Américas ubicada en Santo Domingo de los Tsáchilas año lectivo 2018- 2019; debido a que se observó una disminución del rendimiento académico de los estudiantes, con relación a la cantidad de tareas que realizan diariamente. Esta investigación fue llevada a cabo bajo un enfoque mixto con un diseño no experimental transeccional y un tipo de investigación descriptiva, donde la población se constituyó de 60 individuos y la muestra de 20 estudiantes y 3 docentes. Los instrumentos utilizados para la recolección de los datos fueron una entrevista y encuesta elaborada por las autoras y validada por criterio de expertos. Dando como resultado que la mayoría de los estudiantes y docentes son conscientes de la importancia de la funcionalidad de las tareas en relación con el rendimiento académico.

PALABRAS CLAVE: Rendimiento escolar; Motivación; Educación.

\footnotetext{
${ }^{1}$ Licenciada en Docencia y Gestión de Educación Básica de la Pontificia Universidad Católica del Ecuador Sede Santo Domingo. Docente de la Unidad Educativa Liceo de las Américas. Pontificia Universidad Católica del Ecuador sede Santo Domingo, Santo Domingo,

${ }^{2}$ Licenciada en Docencia y Gestión de Educación Básica de la Pontificia Universidad Católica del Ecuador Sede Santo Domingo. Docente de la Unidad Educativa Genesaret. Pontificia Universidad Católica del Ecuador sede Santo Domingo, Santo Domingo, Ecuador

${ }^{3}$ Doctorando de Ciencias de la Educación. Magister en Gerencia en Liderazgo Educativo, Docente de la Escuela de Ciencias de la Educación de la Pontificia Universidad Católica del Ecuador Sede Santo Domingo. Santo Domingo, Ecuador.

${ }^{4}$ Maestrante en Pedagogía con Mención en Educación Técnica y Tecnológica. Licenciado en Docencia y Gestión de Educación Básica. Miembro del grupo de investigación OGMIOS. Técnico de Gestión Académica de la Pontificia Universidad Católica del Ecuador sede Santo Domingo. Santo Domingo, Ecuador.
} 
Marjorie Narcisa Cevallos Rosero, Tania Estefanía Aguirre Espinosa, Edgar Efraín Obaco Soto...

\section{IMPLICACIONES DE LAS TAREAS ESCOLARES EN EL RENDIMIENTO ACADÉMICO EN LOS ESTUDIANTES DEL SÉPTIMO AÑO DE EDUCACIÓN GENERAL BÁSICA}

\section{ABSTRACT}

The purpose of the present The study The analysis of the impact The work in the place The work in the field The time in the academic field in the center of the educative education Basic of the Educational Unit Liceo de las Américas located in Santo Domingo de los Tsáchilas year school 2018-20199; because it has been observed that students decrease school performance due to the excessive amount of tasks they may have to perform. This research was carried out under a mixed approach with a non-experimental design and a type of descriptive research, where the population refers to 60 individuals and the sample of 20 students and 3 teachers. The instruments used to collect the data were an interview and a survey prepared by the authors and validated by experts. Resulting in the fact that the majority of students and teachers are the importance of the functionality of the tasks in relation to academic performance.

KEYWORDS: School Performance; Motivation; Education.

\section{INTRODUCCIÓN}

La educación es un proceso de socialización que se apoya en diversos elementos para la consecución de destrezas y objetivos que se plantean a nivel general y específico; en donde se necesitan de actividades en clase, trabajos cooperativos, colaborativos, individuales, etc. Entre los trabajos que se considera que se utilizan para la retroalimentación y el aprendizaje individual o por descubrimiento, están las tareas escolares, que han sido objeto de discusión por diversos autores en el siglo actual, debido al mal sentido que se le ha dado a dichas tareas (González y Núñez, 2005).

De acuerdo a las propuestas presentadas por la OMS (2016) a través de la Organización de las Naciones Unidas (ONU), se han realizado peticiones para que se elimine el componente "tareas" de la educación general básica, debido a que es considerada como el elemento que resta el interés al proceso de enseñanza-aprendizaje y lo convierte en un acto rígido. Por otro lado, las razones que plantean las instituciones internacionales son que, en la mayoría de los casos, para la realización de las tareas, influye el aspecto familiar, es decir, que los estudiantes no tienen suficiente autonomía para realizar sus tareas por propia cuenta, lo que conllevan a que estos fracasen en las clases.

Por otro lado, estudios realizados a nivel de Latinoamérica por Suarez, Núñez, Vallejo, Cerezo, Regueiro y Rosario (2014) mencionan que las tareas mejoran las habilidades y capacidades de estudio de los infantes y les enseña que el proceso de aprendizaje no sólo se debe producir en las instalaciones del colegio, sino que se puede generar nuevos conocimientos de manera autónoma, favoreciendo la auto-preparación del alumno; sin embargo, estas suelen presentar resistencia en los estudiantes, debido a que se las considera tediosas, aburridas o "estresantes".

Es así que, se abre la discusión acerca de que, si las tareas escolares son esenciales para el desarrollo del correcto rendimiento escolar, frente a la serie de emociones y actitudes negativas que generan frente a estudiantes y padres de familia; entorno a la necesidad evidente, frente al estudio de la implicación de las tareas en el rendimiento escolar, se han desarrollado una serie de estudios que aclaran el problema desde diversas perspectivas, a continuación, enlistaremos algunos de ellos. 
Un estudio correlacional, con enfoque mixto, elaborado por Rosario, Mourao, Baldaque, Nunes, Núñez, Gonzales-Pineida, Cerezo y Valle (2011), acerca de la relación entre las tareas para la casa (TPC) y el rendimiento final, denominado "Uso de diarios de tareas para casa en el inglés como lengua extranjera (...)" con la finalidad de argumentar la influencia positiva de las tareas en el rendimiento escolar, obtuvo como resultado que el efecto de las TPC sobre el rendimiento es indirecto, es decir, las tareas para la casa sirven de fortalecimiento cognitivo de aprendizajes adquiridos, esta conclusión aportó al presente estudio con el planteamiento de la funcionalidad de las tareas "Refuerzo del aprendizaje" y la consideración del mismo como indicador para la presente investigación, dada la incongruencia determinada por los autores.

Por otro lado, se propone el estudio planteado por Rosario, Mourao, Baldaque, Nunes, Núñez, Gonzales-Pineida, Cerezo y Valle (2009) titulado como "Tareas para casa, autorregulación del aprendizaje y rendimiento en matemáticas" con una metodología cualitativa, a partir de un estudio de casos, buscando analizar la influencia de las tareas para la casa desde la perspectiva estudiantil, en relación con la motivación para la mejora del rendimiento escolar, se concluyó en que: las variables relacionadas con las tareas para la casa, la cantidad, el tiempo, la dificultad, la satisfacción y el esfuerzo obtenido, no inciden directamente sobre el rendimiento de las matemáticas, pero si indirectamente desarrollando habilidades como "la responsabilidad, autoeficacia y desarrollo de hábitos de aprendizaje". Estos resultados aportan a incluir el indicador de desarrollo de habilidades y destrezas para estudiar en la muestra del presente estudio y observar los resultados en el contexto de la realidad educativa ecuatoriana.

Por otro lado, encontramos a Regueiro, Suárez, Valle, Núñez, Rosário (2015) quienes en su trabajo "La motivación e implicación en los deberes escolares a lo largo de la escolaridad obligatoria" de enfoque cuantitativo, con una metodología de análisis multivariado de covarianza, buscando determinar la motivación intrínseca y extrínseca que determina el interés a los deberes, se obtuvo como resultado que, existen diferencias estadísticamente significativas, en función del curso, en la motivación e implicación en los deberes y el rendimiento académico, es decir que, la motivación para la elaboración de las tareas dependen del nivel de complejidad y de la cantidad.

Desde lo antes expuesto, todos los autores se enfocan en el análisis de las tareas escolares relacionadas con diferentes ámbitos de los estudiantes, como, por ejemplo, la motivación, el desarrollo de valores, el refuerzo de áreas, pero no se indaga en la implicación en general de las tareas en el rendimiento escolar, visto desde el ámbito cualitativo y cuantitativo, es decir, la apropiación de competencias reflejadas en las notas.

Por lo cual, con base en toda la problemática y los estudios antes realizados, se desarrolló el presente estudio, teniendo como objetivo general: Analizar el impacto de las tareas escolares en el Rendimiento Académico en los estudiantes de séptimo año de Educación General Básica de la Unidad Educativa Liceo de las Américas ubicada en Santo Domingo de los Tsáchilas año lectivo 2018- 2019.

\section{DESARROLLO}

\section{CONTENIDO}

\subsection{Las tareas escolares}

En el campo educativo, cabe resaltar que los subniveles educativos han dado paso a poner distancias en lo que respecta el contenido. Es por ello que, con cada sub nivel educativo se plantea una serie de tareas las mismas que, buscan vincular resultados ante actividades enviadas a casa 
Marjorie Narcisa Cevallos Rosero, Tania Estefanía Aguirre Espinosa, Edgar Efraín Obaco Soto...

para un eficaz resultado. Según Torres y Yela citados en Quispe (2007) "La tarea debe definirse como una actividad escolar para ayudar al alumno en su trabajo autónomo" (p.36), por otro lado, Montaño (2017) afirma que la tarea escolar es entendida como la recreación de procesos, productos y sujetos implicados en la actividad que permiten a los estudiantes alcanzar metas y objetivos en beneficio de un bien común.

Es por ello que el Ministerio de Educación del Ecuador a partir del 2016, plantea que las tareas escolares sean significativas, en base a este criterio se han clasificado en tres tipos: las asociadas a la lectura, las asociadas a la investigación y las asociadas a la resolución de problemas. Y para que los estudiantes realicen de forma autónoma las tareas que son asignadas por el docente, estos deben haber tenido una adquisición previa del conocimiento, según Carranza y Caldera (2018) la integración de los nuevos conocimientos en la estructura cognitiva de quien aprende supone ciertas condiciones, a saber, la presencia de ideas previas para relacionar el conocimiento previo con el nuevo" (p.75). Porque es importante asociar las experiencias y conocimientos previos con las nuevas teorías del conocimiento.

El significado de tarea tomado de La Real Academia Española (2018) manifiesta que tarea es un: "trabajo a realizarse en un límite de tiempo o es un deber a cargo de los estudiantes, emitidos por los maestros", pero esto hace referencia a cada uno de los actores de la Comunidad Educativa, estudiantes, docentes y padres de familia. Pues para cada uno de ellos tiene el mismo objetivo, sin embargo, le dan un sentido diferente.

En conclusión, la tarea escolar es un proceso de aprendizaje que se lo realiza en un contexto no formal, y que su función relativa es cumplir con el refuerzo del tema impartido en la jornada educativa, y que esta a su vez cumple con requisitos. Cooper en Pan, Irene, Regueiro, Bibiana, Ponte, Beatriz y otros, citado por García, (2016) manifiestan que las tareas enviadas por los profesores para realizarlas fuera del horario escolar, tienen como finalidad que las habilidades y conocimientos adquiridos puedan transferirse a otros ambientes. Por ende, las tareas escolares son un trabajo que los docentes asignan a sus estudiantes para complementar las destrezas adquiridas en clase, fuera del horario escolar.

\subsection{Componentes de las tareas escolares.}

Sabemos que el Proceso de Enseñanza-Aprendizaje (PEA) tiene una gran vinculación con las tareas escolares, pues su objetivo es ayudar al estudiante a concretar los temas trabajados en clases y reforzar el mismo. Pasek y Matos citado por Díaz, Narvaez y Villota (2014), manifiestan que para que una tarea sea adecuada y cumpla los objetivos debe tener los siguientes componentes:

\subsection{Elementos que configuran una tarea escolar}

Toda tarea escolar debe ser eficiente, tener un propósito predeterminado, para lo cual debe cumplir con ciertas normas. Estas normas a seguir están conformadas por contenidos y por procesos cognitivos. Es decir, todas las tareas escolares tienen una vinculación con los materiales y contenidos los cuales se relacionan con objetivos de cada asignatura, así mismo implica habilidades tales como, asignar, memorizar, relacionar, recopilar, entre otras más.

Los elementos de una tarea escolar siempre tienen presente al estudiante y al docente, pues para el estudiante la tarea escolar debe ser un desafío interesante, contener sus respectivos conocimientos 
previos, y desarrollar sus capacidades al límite, es ahí donde va a saber que el esfuerzo que le dedique será el resultado de una tarea bien trabajada.

Por otro lado, los materiales y el contenido de cada tarea escolar deben tener una relación con los conocimientos y con su experiencia en la vida diaria para que este tenga un carácter significativo, es decir no algo estructurado.

\subsection{Tareas apropiadas para cada momento de aprendizaje}

Al crear tareas escolares se debe considerar algunos aspectos como: el contenido que se va aprender, el proceso cognitivo que se desea desarrolla, el estilo de un pensamiento crítico y por último las competencias. Por ello se requiere una planificación con anticipación y no algo improvisado, ya que esto a la larga desmotiva tanto al estudiante como al docente.

En este sentido se debe planificar estrategias distintas que desarrollen diferentes pensamientos, para resolver un problema debemos explicar el paso a paso de cómo resolver el mismo, hacer que el estudiante realice actividades por descubrimiento tiene una esencia magnifica y un resultado tenaz para su transferencia.

Por otro lado, una tarea esencial debe introducir al estudiante, en diferentes actividades ya sean estas individuales o en grupos, de tal manera que él sea el protagonista, esto le permitirá a él desarrollar al máximo su potencial en todos los ámbitos.

\subsection{Tipos de tareas escolares}

Al igual que existe una gran diversidad de estilos de aprendizaje es conveniente tomar en cuenta los diferentes tipos de tareas escolares, por esta razón, López citado por García (2016) manifiesta que no existe en realidad algún tipo de tarea. Yvonne citado por Posada y Taborda (2012) contradice lo afirmado por López, ya que manifiesta que las tareas se dividen en dos grupos: Las de prácticas que son aquellas que se enfatizan en reforzar conocimientos recién adquiridos y en potenciar aún más sus habilidades, estas adquieren más valor cuando son evaluadas por el Docente ya que ahí el estudiante tomará en cuenta en que falló y donde debe reforzar.

Además, tenemos las de preparación, estas se encargan de inmiscuir al estudiante en el tema que se va abordar en la clase, para aplicar esta tarea existen actividades como buscar información en línea sobre el significado de palabras claves o actividades didácticas de acorde a la edad. Es así que Fernández y Rivero (2014) aseguran que la implementación de las TIC’S en los salones de clase permite potencializar el uso de entornos virtuales de aprendizaje.

\subsection{Características de las tareas escolares.}

Para que una tarea ayude realmente al desarrollo de los niños debe ser acorde a su edad, que tenga sentido con el tema tratado y principalmente que tenga un propósito, a su vez debe tener sus especificaciones claras, es así como va ayudar a enriquecer los conocimientos del niño y desarrollar al máximo sus aptitudes. Carrobles citado por Montuy (2012) manifiesta que las tareas mal elaboradas no ayudan a potenciar habilidades del alumno, pues estos las realizan ya sea por conseguir una calificación, o también se limitan a ser una repetición de lo trabajado ya en el aula.

También, suelen ser mecánicas, no son claras, no tienen una supervisión en casa, son fatigosas o simplemente se limitan a que el docente debe cumplir con un cronograma de actividades establecidos en la Unidad Educativa y en las planificaciones presentadas. Cooper, Cosden, Morrison, Albanese y Macias citados por Valle, Pan, Núñez, Rosário, Rodríguez y Regueiro (2015) consideran "los deberes escolares como una parte más del trabajo académico del alumnado, que se 
Marjorie Narcisa Cevallos Rosero, Tania Estefanía Aguirre Espinosa, Edgar Efraín Obaco Soto...

asignan para realizar fuera del periodo habitual de clases con el fin de extender y ampliar la práctica de habilidades académicas" (p.562).

\subsection{Los actores que intervienen en la realización de tareas: Padres de familia y estudiantes}

Las tareas escolares enviadas a casa pueden ser consideradas por los maestros como una práctica que les permite a los alumnos fortalecer los conocimientos trabajados en clase, les ayuda a formar hábitos de estudio, enfocarse en realizar un trabajo independiente y responsable, desarrollar su pensamiento crítico y ser el principal protagonista de su propio aprendizaje.

Los padres cumplen un papel muy importante porque ellos son los primeros guías de sus hijos y la ayuda complementaria. De acuerdo al nivel educativo que plantea Eggers (2015), el Laboratorio Latinoamericano de Evaluación de la Calidad de la Educación demostró la importancia que tienen la formación de los padres y la disponibilidad de libros en el hogar, con la tasa de avance que logran los estudiantes.

El nivel académico de los padres juega un papel importante, ya que pueden ayudar a sus hijos en las tareas que se envían fuera del horario escolar, pero tiene la parte negativa, ya que, a ser personas con preparación académica, no tienen tiempo para sus hijos, es por ello la importancia de dosificar las tareas, y enviar cosas puntuales que el estudiante en su gran mayoría pueda realizarlas solo. La vigilancia de un adulto responsable solo se limitará al cumplimiento de las mismas.

Para Philippe Meirieu citado por Posada y Taborda (2012), manifiesta que las tareas que se realizan en casa contribuyen de una forma sistemática a las desigualdades sociales, familiares y del entorno, pues quien goza de un contexto social favorable y un entorno familiar enriquecido por lo general tendrá la ventaja sobre aquel que no lo tiene o que no goza de ciertas comodidades.

Los docentes en el aula deben reconocer el valor que le otorgan a la tarea, ya que es una incertidumbre saber quién fijo el cocimiento, si los padres de familia o los alumnos. Según Cartledge y Sasser citado por Posada y Taborda (2012) los docentes son los encargados de descubrir una estrecha relación entre las tareas escolares y el rendimiento académico pues nadie apostaba en esta relación directa.

\subsection{Rendimiento Académico}

El rendimiento escolar es un concepto que requiere de un análisis contextualizado para comprender su impacto el proceso educativo. En la parte académica, Navarro (2003) explica que la habilidad no es sinónimo de esfuerzo, debido a que, en varios de los casos, los docentes valoran más el esfuerzo que la habilidad. A partir de lo mencionado, el rendimiento académico tendría que ver con la actitud y no con la aptitud al momento de realizar una actividad, siendo esto, uno de los principales errores en las aulas de clases.

Desde la perspectiva de Morales, Morales y Olguín (2007) el rendimiento académico no es la simple acumulación de conocimientos y los resultados satisfactorios de una evaluación, va más allá de esto y, se relaciona con la calidad de la educación, puesto que, el rendimiento individual del estudiante tendrá relación con sus calificaciones y el nivel de conocimiento que tiene al momento de realizar actividades.

Por lo antes mencionado, hablar de rendimiento académico en varias ocasiones suele solo enfocarse en una calificación antes que en el aprendizaje de los estudiantes. Para poder palpar el ritmo de aprendizaje, se denota una calificación como lo manifiesta González y Núñez (2005), además, las medidas del rendimiento se obtienen a partir de las notas de los alumnos con base a puntajes y

92 UNESUM-Ciencias. Publicación cuatrimestral. Vol. 4, Año 2020, No. 1 (Enero - Abril) 
escalas previamente establecidas, pero con la salvedad de que existen actividades previas que los estudiantes van resolviendo conforme a su aprendizaje.

Con respecto a lo mencionado, Jiménez (2000) manifiesta que el rendimiento académico hace referencia al nivel saberes que poseen los estudiantes para desempeñarse en cualquier área, teniendo en cuenta la edad y el nivel académico en el que se encuentra; sin embargo, esto se ve influenciado también por el contexto en el que se educan los estudiantes, ya que no todos tienen la misma motivación, recursos o ayuda para lograr el máximo potencial.

Por otro lado, González y Núñez (2005) mencionan que la ponderación que se dé a los trabajos, actividades, tareas, ya sean dentro o fuera del salón, se los hace con la finalidad de verificar cuanto aprendió el estudiante al momento de realizar las actividades establecidas y, en caso que no haya trabajado, seguramente la calificación será baja. Este es el malestar que muchos padres de familia perciben, pero lastimosamente el fin no es obtener una buena calificación, más bien es obtener lograr criterios de aprendizaje que a un futuro se puedan evidenciar el éxito de los estudiantes.

A partir de la investigación y la recogida de datos en el contexto educativo, se puede analizar el rendimiento académico de los estudiantes, ante esto, Guerrero (2009) pudo determinar que las asignaturas de Matemáticas son las que más incumplimientos de tareas presentan entre 192 estudiantes evaluados, como se puede ver en la tabla 2; sin embargo, este autor no solo se centra en las calificaciones, sino en cómo se las lograron, siendo el cumplimientos de las tareas escolares un factor de gran influencia.

\subsection{Tareas enviadas a casa y tareas en la escuela}

Actualmente, los estudiantes tienden a ver las tareas como un reto, ya sea por el tiempo que se estime a cada de ellas, mencionan Travé, Estepa y Delval (2017) que "Un pensamiento práctico, reflexivo y crítico para contribuir a mejorar la realidad socioeconómica; y, en cuanto a los aspectos psicológicos, promuevan un aprendizaje significativo y relevante relacionando las ideas personales y los intereses del alumnado con las nuevas informaciones" (p.332) no todas las tareas se deben hacer en el texto, el aprendizaje debe ser practico, y relacionarlo con otras actividades no debe estar desvinculada la escuela ni de la vida diaria.

De acuerdo a los estudios realizados por Xu citado por Suárez, et al (2012) menciona que algunos estudiantes para hacer un mejor trabajo en casa acuden a la ayuda de sus progenitores o encargados adultos y es allí donde ellos cumplen un papel protagónico, saber organizar el tiempo evitando que los estudiantes se distraigan es primordial, hasta el afecto por parte de los progenitores es un punto clave para el buen desempeño en la etapa escolar, los hogares bien estructurados tienen reglas y son exigentes con sus hijos y se ha comprado que aquellos estudiante llevan un orden y un aprendizaje adecuado no solo en la escuela sino fuera del horario escolar.

Cabe resaltar que los padres juegan un papel muy importante en el campo educativo, ya que son quienes pasan más tiempo en sus hogares y deben ser quienes supervisen el trabajo realizado por sus hijos tanto en la escuela como en casa. Es por ello que Corno citado por Suárez, et al (2012) manifiesta sobre las tareas, que los estudiantes deben hacer uso y dominio de su tiempo y desde edad temprana adquirir la responsabilidad del tiempo en cuanto a sus tareas fuera del horario escolar, ya que ellos ayudan a desarrollar las destrezas adquiridas en la escuela, se debe tener un control y sin caer en el juego de pagar el esfuerzo con grandes recompensas.

\subsection{Organización escolar}


Marjorie Narcisa Cevallos Rosero, Tania Estefanía Aguirre Espinosa, Edgar Efraín Obaco Soto...

La organización escolar juega un papel decisivo en el desarrollo educativo de sus miembros. A través de ella puede concretarse la construcción de una escuela inclusiva y reductora de desigualdades. González, 2008; Lorente, 2006; Muñoz, Rodríguez y Barrera, 2013 citados en Vásquez (2019) plantean que los modelos de dirección educativa y todos los departamentos y comisiones que existen contribuyen a que los procesos educativos sean ricos y valiosos para todos. Aunque muchas veces esta no es la realidad actual, los entornos poco adecuados maximizan el fracaso escolar, las políticas de estados no garantizan el bienestar estudiantil.

En la actualidad podemos palpar que existen muchas personas que se sienten fracasadas a temprana edad, a su vez se sienten ofuscadas por motivo que no pueden cumplir con una actividad o a su vez porque no se sienten capaces de poder seguir con el estudio. Es por ello que se puede llamar fracaso escolar al no poder culminar el proceso de formación educativa tal cual lo plantea Moreno citado por Vásquez (2016) que el padre de familia, el docente y la sociedad, entra jugando un papel importante en lo educativo ya que por diversas circunstancias los estudiantes deciden alejarse de lo realmente importante.

Pero no se puede determinar en sí que factor hizo que los estudiantes dejen de lado al estudio si son los factores internos o externos. Por ende, el docente es el primero que debe manifestar si el estudiante muestra poco interés en lo académico, ya que gran parte del día permanece allí, y mantener una adecuada comunicación para poder fomentar nuevamente el interés por la culminación de sus estudios.

La investigación posee una metodología orientada bajo un enfoque mixto, por tal razón, se realizó un proceso de recogida de datos cualitativos y cuantitativos para la propuesta de una interpretación y emisión de juicios críticos sobre las implicaciones de las tareas escolares en el rendimiento académicos de los estudiantes. El diseño de investigación que se consideró para el estudio es de tipo no experimental, ya que no se realizó una manipulación de variables. Debido al tipo de diseño, se aplicó la investigación transeccional, Por otro lado, el tipo de investigación que se aplicó es descriptivo porque según Tamayo y Tamayo (2004) permite la descripción y análisis mediante la observación de un fenómeno.

Se utilizó un muestro no probabilístico de tipo intencional, en donde la población se centra en 60 estudiantes de séptimo año de la Unidad Educativa Liceo de las Américas, de acuerdo a lo mencionado, la muestra se conformó de 20 estudiantes de séptimo año de EGB paralelo A, además de 3 docentes que imparten clases en estos años de EGB.

Para la recolección de la información, los instrumentos que se propusieron para el estudio fueron aplicados en un solo momento, el cual fue en el primer parcial del segundo quimestre en las aulas de séptimo año de Educación General Básica de la Unidad Educativa Liceo de las Américas. La técnica e instrumentos de recogida de datos fueron la entrevista, la cual se aplicó para analizar la opinión de los docentes, respecto a las tareas que se envían y si estas influyen en el rendimiento académico.

Por otro lado, la encuesta permitió analizar las opiniones de los estudiantes de séptimo año de EGB de la Unidad Educativa Liceo de las Américas, sobre la influencia e implicaciones de las tareas en el rendimiento académico. Finalmente, como técnica de análisis de datos, la primera corresponde a la interpretación cualitativa de las respuestas que dieron los docentes en la entrevista; por otro lado, la segunda técnica permitió, esquematizar en tablas y figuras los datos estadísticos de la encuesta a través Statistical Package for the Social Sciences (SPSS) para su posterior análisis e 
interpretación. Y finalmente los resultados fueron triangulados para una mejor comprensión y elaboración de la discusión.

\section{RESULTADOS}

\subsection{Primer resultado:}

Tabla 1

Tabla de consistencia lógica

\section{Preguntas de la entrevista}

Análisis

1. ¿Considera que son necesarias las tareas enviadas a casa?

R1: Sí, porque es una actividad que refuerza el conocimiento académico.

R2: Sí, porque crea hábitos de estudio para la vida posterior

$\mathrm{R} 3$ : $\mathrm{Si}$, porque los estudiantes se hacen responsables

de su propio aprendizaje.

$\mathrm{R} 4: \mathrm{Si}$, porque hacen que se involucren de forma autónoma.

2. ¿Cuáles son las características de una tarea para que haya un impacto positivo en los estudiantes y su aprendizaje?

R1: Propósito específico

R2: Enriquecer los conocimientos

R3: Instrucciones claras.

R4: Desarrollar aptitudes del estudiante.

3. Teniendo en cuenta la complejidad de las tareas que orienta, ¿Cuánto tiempo emplean los estudiantes para concluirla?

$\mathrm{R} 1: 20$ minuto

$\mathrm{R} 2: 20$ minuto

R3:20- 40 minutos

$\mathrm{R} 4: 20$ - 40 minutos

4. ¿De qué forma usted despierta el interés de sus estudiantes para que realicen la tarea?

$\mathrm{R} 1$ : Que contengan experimentos.

R2: Situaciones reales

R3: Poco trabajo

R4:Motivaciones en el aula

5. ¿Con cuánto tiempo de antelación envía las tareas a sus estudiantes generalmente?

$\mathrm{R} 1$ : Entre 4 y 6 días

R2: Entre 3 y 4 días

R3: Entre 3 y 4 días

R4:Entre 3 y 4 días
A2: En la pregunta número 2, las respuestas son diferentes, por lo tanto, se menciona que las características de una tarea pueden ser sobre un propósitos, conocimientos, instrucciones o aptitudes.

A3: En la pregunta número 3, se determina que el tiempo empleado para realizar las tareas por los estudiantes está alrededor de los 20 y 40 minutos; dependiendo del nivel de complejidad de las mismas

A4: En la pregunta número 4, las docentes explican que las tareas son más interesantes cuando contienen experiencias concretas y no son extensas.

A5: En la pregunta número 5, se detalla que los docentes envían las tareas con un tiempo de antelación que comprende de 3 a 6 días. 
Marjorie Narcisa Cevallos Rosero, Tania Estefanía Aguirre Espinosa, Edgar Efraín Obaco Soto...

\author{
6. ¿Indique qué tipo de tareas envía con más \\ frecuencia a sus estudiantes? \\ R1: Ejercicios del libro de texto \\ R2: Trabajos en grupo \\ R3: Búsqueda de información \\ $\mathrm{R} 4:$ Trabajos en grupo
}

\section{7. ¿Las tareas en la EGB deberían incrementarse, mantenerse, disminuir 0 desaparecer?}

R1: Dosificarse, debido al nivel de complejidad de algunas.

R2: Mantenerse, porque está bien el sistema

educativo.

R3: Mantenerse.

R4: Mantenerse pero seguir la guía de sugerencias

del Ministerio de Educación.
A6: En la pregunta número 6, los docentes mencionan que los tipos de tarea más frecuentes que envían tienen que ver con procesamiento, análisis y aplicación de información y trabajos en grupo.

A7: En la pregunta número 7, los docentes coinciden en su mayoría en que las tareas deben mantenerse por que las disposiciones del ministerio de educación son correctas, pero también se menciona que deben dosificarse en función del nivel de complejidad.

\section{Conclusiones:}

En la pregunta número 1, todas las respuestas afirman que las tareas para la casa son necesarias, mencionando que los propósitos son: refuerzo de conocimientos adquiridos, creación de hábitos de estudio, formación de valores en los estudiantes y fomento de la autonomía personal.

En la pregunta número 2, las características que establecen los docentes entrevistados para que las tareas enviadas para la casa tenga un impacto positivo en su aprendizaje son: ha de tener un propósito específico, dado a que, si se cae en generalidades, el estudiante podría desviarse de lo que realmente se busca; tiene que enriquecer los conocimientos, es decir, con base en lo que ya sabe, crear nuevas estructuras cognoscitivas más duraderas; poseer instrucciones claras, debido a que si no se detalla lo que se necesita que el alumno realice, pueden haber desviaciones en el desarrollo.

En la pregunta número 3, se expone que los docentes coinciden que el tiempo adecuado para concluir las tareas, en función de la complejidad, es de 20 minutos 40 minutos, debido a que, es el tiempo en que el estudiante puede mantenerse concentrado y rendir la máxima capacidad.

En la pregunta número 4, se muestra que los docentes despiertan el interés por las tareas a través de actividades que tengan que ver con la aplicación de todos los sentidos, es decir, experiencias concretas; aplicando experimentos y situaciones que lleven al estudiante a relacionarse con el mundo sensible, así mismo, que exista una motivación previa por parte del docente y que no sean excesivas en cantidad.

En la pregunta número 5, se muestra que, los docentes consideran como el tiempo adecuado de plazo para realizar las tareas el intervalo de 3 a 6 días, debido a que, si se pretende realizar tareas motivadoras, que posean relación con los sentidos, construcción y experimentación con el mundo real, se requiere un amplio plazo temporal.

En la pregunta número 6, los docentes aclaran que las tareas que se envían con mayor frecuencia están en función de conocimientos conceptuales y procedimentales, aplicando habilidades de búsqueda, análisis, síntesis y producción, así mismo, trabajos que requieran la interacción entre varias personas.

En la pregunta número 7, se determina que en la educación general básica, las tareas para la casa deben mantenerse, porque las consideraciones del Ministerio de Educación del Ecuador son correctas, dada a la investigación previa y la fundamentación en currículos extranjeros; pero dosificándose en función de la complejidad.

Nota: R (Respuesta); A (Análisis). Fuente: Elaborado por Cevallos, M. \& Aguirre, T.

\subsection{Segundo resultado}

Tabla 2

Tabla del análisis de los indicadores de la encuesta

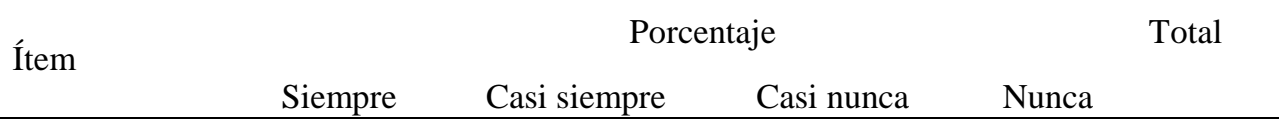




\begin{tabular}{cccccc}
\hline $\begin{array}{c}\text { Cumplimiento de tareas para } \\
\text { la casa }\end{array}$ & $65 \%$ & $35 \%$ & & $100 \%$ \\
$\begin{array}{c}\text { Consideración acerca de si las } \\
\text { tareas mejoran el rendimiento } \\
\text { escolar }\end{array}$ & $30 \%$ & $55 \%$ & $15 \%$ & $100 \%$ \\
$\begin{array}{c}\text { Necesidad de adultos para } \\
\quad \text { resolver las tareas }\end{array}$ & & $25 \%$ & $65 \%$ & $10 \%$ & $100 \%$ \\
$\begin{array}{c}\text { Ayuda de las tareas para la } \\
\text { comprensión de contenidos } \\
\text { que se trabajan en clase }\end{array}$ & $45 \%$ & $40 \%$ & $15 \%$ & & $100 \%$ \\
$\begin{array}{c}\text { Percepción de la cantidad } \\
\text { excesiva de tareas para la casa } \\
\begin{array}{c}\text { Claridad en las instrucciones } \\
\text { al enviar tareas para la casa }\end{array}\end{array}$ & $35 \%$ & $25 \%$ & $60 \%$ & $15 \%$ & $100 \%$ \\
$\begin{array}{c}\text { Uso de material didáctico para } \\
\text { impartir clases }\end{array}$ & $3 \%$ & $65 \%$ & & & $100 \%$ \\
\hline
\end{tabular}

Nota: Fuente: Elaborado por Cevallos, M. \& Aguirre, T.

\section{Análisis e interpretación:}

Los datos de la encuesta demuestran que el cumplimiento de las tareas posee un porcentaje alto en la escala planteada, con el $65 \%$ en siempre y $35 \%$ en casi siempre, demostrando que los estudiantes son responsables en la realización de las tareas para la casa, así mismo, otro resultado alto que se orienta al siempre (con el 45\%) y "casi siempre" (con 40\%) es la comprensión de contenidos que se trabajan en la clase, a partir de las tareas escolares, lo que demuestra que las tareas escolares, en sentido de desarrollo de competencias, es útil, desde la perspectiva estudiantil, así mismo, otros indicadores en los que se obtuvieron porcentajes positivos fueron: la claridad de instrucciones para la tarea(35\% en siempre y $65 \%$ en casi siempre) exponiendo que el docente está realizando su trabajo de manera adecuada en la orientación de las tareas, facilitando el cumplimiento de las tareas; y la consideración, desde la perspectiva estudiantil, acerca de si las tareas para la casa mejoran el rendimiento escolar, desde datos numéricos y la adquisición de competencias, en la que se evidencia que el 30\% de la población encuestada mencionó que siempre, el 55\% que casi siempre y el $15 \%$ que nunca.

Por otro lado, los resultados con porcentajes orientados mayormente a la escala nunca y casi nunca, fueron los indicadores: necesidad de adultos para resolver las tareas, donde se presenta que el $60 \%$ mencionó que casi nunca necesitan de adultos, lo que refleja independencia en los estudiantes, pero podría significar también la falta de acompañamiento familiar; y la percepción acerca de si las tareas enviadas para la casa son excesivas, donde el $60 \%$ de los estudiantes contestaron que, casi nunca se envían tareas excesivamente.

\subsection{Segundo resultado}

Tabla 3

Ponderación de los promedios del primer parcial.

\begin{tabular}{lllll}
\hline \multicolumn{1}{c}{ Escala } & & Frecuencia & Frecuencia relativa & Porcentaje \\
\hline DAR $(9-10)$ & 9 & & 0.39 & $39 \%$ \\
AAR $(7-8.99)$ & 14 & & 0.61 & $61 \%$ \\
\hline
\end{tabular}


Marjorie Narcisa Cevallos Rosero, Tania Estefanía Aguirre Espinosa, Edgar Efraín Obaco Soto...

\begin{tabular}{llll}
\hline PARA $(4.01-6.99)$ & 0 & 0 & 0 \\
NAAR $(<4)$ & 0 & 0 & 0 \\
Total & 23 & 1.00 & $100 \%$
\end{tabular}

NOTA: DAR (Domina los aprendizajes requeridos); AAR (Alcanza los aprendizajes requeridos); PARA (Próximo a alcanzar los aprendizajes requeridos); NAAR (No alcanza los aprendizajes requeridos).

\section{Analisis e interpretación:}

De los resultados obtenidos en las calificaciones de los estudiantes de séptimo grado de la Unidad educativa Liceo de las Américas (ver Aenxo 5) se descubrio que el 39\% de los estudiantes se encuentran en una escala del DAR y el $61 \%$ en AAR, lo que indica que el grupo de estudiantes se encuentra con un rendimiento académico alto. Dado que los resultados que se presentan son la recopilacion de tareas grupales, individuales, y tareas de casa. Por la tanto, se puede determinar que el tipo de tareas extracademicos estan siendo favorables para todo el grupo en el sentido sumativo, con una función de promocion que se caracteriza por el uso de una adecuada metodologia que pemrita alcanzar los aprendizajes requeridos.

\section{DISCUSIÓN}

Los docentes mencionan que las tareas son necesarias para fortalecer el aprendizaje, siempre y cuando se orienten al refuerzo de conocimientos y motiven el interés de los estudiantes, lo que posee relación con los resultados de los autores Rosario, Mourao, Baldaque, Nunes, Núñez, Gonzales-Pineida, Cerezo y Valle (2011), quienes concluyen su estudio mencionando que las tareas son importantes en función del refuerzo que represente para el estudiante, y el aprendizaje nuevo, que representa el elemento motivador, que se puede obtener, esto expone que las tareas escolares tendrán un impacto, cuando se las relacionen con conocimientos ya adquiridos y sirvan para adquirir a nuevos conocimientos.

Los docentes mencionan que las tareas deben tener instrucciones claras y especificaciones; por lo tanto, de acuerdo a la complejidad de las actividades estas pueden ir desde los 20 a los 40 minutos de trabajo, es decir, no deben poseer cantidad excesiva de trabajo, lo que concuerda con el postulado de los autores, Regueiro, Suárez, Valle, Núñez y Rosário (2015), quienes aclaran que la motivación que los estudiantes tengan para desarrollar las tareas, que condicionará el rendimiento, es dependiente al nivel de complejidad; ya antes mencionado, y a la cantidad, esto recalca la importancia de que una tarea no exceda un tiempo determinado, consiguiendo así el mejor rendimiento que el estudiante pueda ofrecer, en la temporalidad máxima de concentración.

A pesar de que, las tareas no superan los 40 minutos de actividad, los docentes explican que los intervalos de envío deben ser entre 3 y 6 días de diferencia, esto se lo propone de acuerdo a la complejidad de la tarea, esto se considera que sucede así, dado a que, se requiere un mayor espacio de días, para poder trabajar el tiempo adecuado, es decir, trabajar por períodos diarios de tiempos cortos, donde el rendimiento sea el máximo; la importancia de ello, lo dejan claro Rosario, Mourao, Baldaque, Nunes, Núñez, Gonzales-Pineida, Cerezo y Valle (2009) determinando en su estudio que la temporalidad y espacio de envío de tareas ha de fluctuar, debido al desarrollo que así se consigue, porque, entre más días tengan para realizar las tareas, se desarrollarán mayores valores, como: la responsabilidad, autoeficacia y desarrollo de hábitos de aprendizaje. 
La dosificación de las tareas es de suma importancia para los docentes, pero lo que consideran relevante es la posibilidad de desarrollar operaciones mentales para la búsqueda de información, el análisis, la síntesis y la producción, en función de que, a partir de lo que se aprendió, se afiancen conocimientos y a su vez, se creen otros. Del mismo modo, mencionaba Posada y Tobarda (2012) acerca de la funcionalidad de las tareas, recalcando que, una tarea pertinente, se desarrolla habilidades de autorregulación, afianzamiento, autoevaluación y producción de nuevos saberes, por lo cual, está claro que no se trata de conseguir una mera nota, sino de desarrollar formativa e íntegramente al estudiante.

Los estudiantes suelen realizar tareas con frecuencia, apoyándose en la idea de que estas son dosificadas y contribuyen al desarrollo cognitivo de los infantes; tal como lo mencionan Carranza y Caldera (2018) en donde la integración de los nuevos conocimientos en la estructura cognitiva de quien aprende supone ciertas condiciones que contribuyen, a saber, la presencia de ideas previas para relacionar el conocimiento previo con el nuevo, favoreciendo el desarrollo de habilidades cognitivas en el trascurso de la vida estudiantil de cada uno de los infantes, creando en su cognición esquemas mentales que permiten un desempeño escolar.

A pesar de que existe un $15 \%$ de estudiantes que no consideran a las tareas como un elemento que fortalece el rendimiento académico, el restante porcentaje de estudiantes muestran interés en hacerlas. La situación que pueden tener ese minoritario porcentaje de estudiantes, posiblemente esté relacionada con la motivación extrínseca que recibe en casa, la cual debe ser fundamental para que se cumplan con las tareas, es así que Regueiro, Suárez, Valle, Núñez, Rosário (2015) mencionar que la motivación es un factor decisivo en el cumplimiento deberes y el rendimiento académico, es decir que, la motivación para la elaboración de las tareas dependen del nivel de complejidad, de la cantidad y del ZDP (Zona de Desarrollo Próximo), en donde debe existir una persona que incentive que se desarrolle dicha motivación por la realización de las tareas.

Por otro lado, existen diversos factores que hacen que los estudiantes se sientan desmotivado al momento de realizar una tarea, como son; la no comprensión de la asignatura, la extensión de las actividades y el tiempo de clases, lo que conlleva a que en muchas ocasiones se realicen tareas de mala calidad; es por ellos que Carrobles citado por Montuy (2012) manifiesta que las tareas mal elaboradas no ayudan a potencializar las habilidades del alumno, pues estos las realizan ya sea por conseguir una calificación, o también se limitan a ser una repetición de lo trabajado ya en el aula. A partir de esta idea, se expone que el $88 \%$ de los encuestados requieren necesariamente de ayuda para cumplir sus tareas de manera eficaz.

Como se lo menciona en la entrevista, el tiempo que le dedican los estudiantes a las tareas no supera los 40 minutos, pero esto se contrasta con utilidad que estas tienen al momento de comprender los contenidos de clases. Es por ello que Corno citado por Suárez, et al (2012) manifiesta que los estudiantes deben hacer uso y dominio de su tiempo; y desde edad temprana adquirir la responsabilidad del tiempo en cuanto a sus tareas fuera del horario escolar, ya que ellos ayudan a desarrollar las destrezas adquiridas en la escuela, se debe tener un control y sin caer en el juego de pagar el esfuerzo con grandes recompensas. Los datos muestran que el $85 \%$ de los estudiantes aprenden más con las tareas, dosificando el tiempo y creando conciencia en la utilización del tiempo en cuanto la realización de cualquier tarea.

Los datos de la entrevista y la encuesta se contrastan, ya que los estudiantes y docentes mencionan que no existe un exceso de tareas para la casa, además el intervalo de envío de tareas corresponde a 1 o 2 veces por semana. Lo que, si queda claro, que las asignaturas de Matemática y Lengua y 
Marjorie Narcisa Cevallos Rosero, Tania Estefanía Aguirre Espinosa, Edgar Efraín Obaco Soto...

Literatura es en donde se envía la mayor cantidad de tareas, además, las actividades que se proponen en su mayoría corresponden a ejercicios del texto del estudiante. Esto se relaciona con lo que manifiesta Cooper citado por Rosario, et al. (2009) afirman que las tareas poseen un potencial increíble de autonomía y responsabilidad, pero en dependencia de la calidad sobre la cantidad y también según Cartledge y Sasser citado por Posada y Taborda (2012) ellos aseguran que las tareas que se envían a casa en su mayoría están relacionadas con ejercicios prácticos.

Como se lo ha mencionado, las tareas no tienen un efecto fatigante en los estudiantes porque no son extensas, por esta misma razón, los estudiantes explican que estas no tienen relación con su rendimiento académico y, sus calificaciones superan los 8 puntos sobre 10. Mencionado resultado está en contra a lo que establece Gonzales (2012) sobre la opinión de los estudiantes sobre las tareas escolares, dado que se comprobó que los alumnos si creen que las tareas son importantes y buenas para ellos. El resultado es contundente, porque el $92 \%$ piensa que si es beneficioso orientar deberes para refuerzo del contenido tratado en la jornada de clases.

Finalmente, los estudiantes mencionan que una de las causas para no hacer las tareas es el exceso (25\%), pero al contrario de esto, existen otras causas (50\%) que no son determinadas, tal es el caso, de la ayuda que reciben en casa, tener los materiales necesarios o la comprensión de las instrucciones de la tarea. Lo mencionado con anterioridad está en concordancia con lo que establece Regueiro, Suárez, Valle, Núñez y Rosário (2015) que la motivación para la elaboración de las tareas va a depender del nivel de complejidad que le asigne el maestro y de la cantidad. Dado que se verá reflejado en su rendimiento académico.

\section{CONCLUSIONES}

Se analizó el grado de cumplimiento de las tareas escolares del séptimo año, mediante la aplicación de una encuesta a los estudiantes y una entrevista a los docentes de la Unidad Educativa "Liceo de las Américas" y a su vez tomamos como referencia los promedios obtenidos de los estudiantes durante el periodo educativo, los cuales arrojaron los siguientes resultados:

Encuesta a los estudiantes: La mayoría de los estudiantes son conscientes de la importancia de la funcionalidad de las tareas en relación con el Rendimiento Académico, además que fomenta la responsabilidad y les refuerza lo que han aprendido en clase, el tiempo que le dedican a las tareas son aproximadamente 30 minutos tres veces por semana y les permite realizar actividades extracurriculares en su gran mayoría las tareas las realizan sin ayuda de un adulto, porque son actividades aprendidas en clase.

Entrevista a los Docentes: se pudo evidenciar que los docentes están de acuerdo en que las tareas son importantes dentro de la unidad educativa, pero se debe regular en cuanto al tiempo y la funcionalidad para fomentar en los estudiantes hábitos de estudios y refuerzo de lo aprendido en clase, y lograr en los estudiantes autonomía al momento de la realización de las tareas, los docentes deben seguir las sugerencias enviadas por el Ministerio de Educación para la asignación de las tareas.

Promedios parciales: en la columna de tareas se evidencia que aquellos estudiantes que cumplieron con las tareas asignadas por sus docentes obtuvieron un buen aprovechamiento que se posiciona en la escala de "dominar los aprendizajes adquiridos" en relación con aquellos que no cumplieron las tareas su promedio se vio afectado y obtuvieron notas que reflejan que alcanza los aprendizajes requeridos.

\section{REFERÉNCIAS BIBLIOGRÁFICAS}


Carranza, R., Caldera, F. (2018). Percepción de los Estudiantes sobre el Aprendizaje Significativo y Estrategias de Enseñanza en el Blended Learning. REICE. Revista Iberoamericana sobre Calidad, Eficacia y Cambio en Educación, 16(1), 73-88. Doi: http://dx.doi.org/10.15366/reice2018.16.1.005

Díaz, A., Narvaez, M. y Villota, M. (2014). El sentido de las tareas escolares en los actores educativos de la institución Madre Caridad en el Municipio de San Juan de Pasto. (Grado). Universidad de Nariño. Colombia. Recuperado de: http://biblÑioteca.udenar.edu.co:8085/atenea/biblioteca/90551.pdf

Eggers, K. (2015). Factores de eficacia escolar asociados al aprendizaje de alumnos del sistema de Telesecundaria en México. (Doctoral). Universidad Complutense De Madrid, España. Recuperado de: https://eprints.ucm.es/40505/1/T38139.pdf

Fernández, A., y Rivero L. (2014). Las plataformas de aprendizajes, una alternativa a tener en cuenta en el proceso de enseñanza aprendizaje. Revista Cubana de Informática Médica, 6(2), 207-221. Recuperado de: http://scielo.sld.cu/scielo.php?script=sci_arttext\&pid=S1684-18592014000200009\&lng=es\&tlng=es.

García, R. (2016). Deberes escolares y rendimiento académico. (Tesis de grado). Universidad De Jaén, España. Recuperado de: http://tauja.ujaen.es/bitstream/10953.1/3027/1/Garca_Daz_Roco_TFG_Educacin_Primaria.PDF.pdf

Gonzales, F. (2012). La opinión de los estudiantes sobre las tareas escolares. Recuperado de: https://www.navarra.es/NR/rdonlyres/D096F1EB-B08C-4554-82D5C402A27850AF/221905/230512ed70estudio.pdf

González, J., y Núñez, J. (2005). La implicación de los padres y su incidencia en el rendimiento de los hijos. Revista de psicología y educación, 1 (1). 115 -134. Recuperado de: http://www.revistadepsicologiayeducacion.es/pdf/9.pdf

Guerrero, T. (2009). La tarea escolar como problemática educativa (Tesis de maestría) Universidad Andina Simón Bolívar, Ecuador. Recuperado de: Guerrero, T. (2009). La tarea escolar como problemática educativa (Tesis de maestría) Universidad Andina Simón Bolívar, Ecuador. Recuperado de: https://bit.ly/2REp1nX

Guerrero, T. (2009). La tarea escolar como problemática educativa (Tesis de maestría) Universidad Andina Simón Bolívar, Ecuador. Recuperado de: http://repositorio.uasb.edu.ec/bitstream/10644/309/1/T737-MGE-GuerreroLa\%20tarea\%20escolar\%20como\%20problem\%C3\%A1tica\%20educativa.pdf

Ley orgánica de educación intercultural. (2008). Presidencia de la República del Ecuador. Recuperado de:https://oig.cepal.org/sites/default/files/2011_leyeducacionintercultural_ecu.pdf

López, I., Regueiro, B., Ponte, B., Rodríguez., S., Piñeiro, I., y Valle, A. (2013). Motivación, implicación en los deberes escolares y rendimiento académico. Aula abierta, 41(3), 13-22. Recuperado de: https://dialnet.unirioja.es/servlet/articulo?codigo $=4401103$. https://dialnet.unirioja.es/servlet/articulo?codigo $=4401103$

Ministerio de Educación (2016). Guía de sugerencias de tareas escolares. Recuperado de: https://educacion.gob.ec/wpcontent/uploads/downloads/2017/01/guia_sugerencias_tareas_2016.pdf

Montaño., P. (2017). Las tareas una estrategia de activación del pensamiento. Recuperado de: http://www.quadernsdigitals.net/datos/hemeroteca/r_24/nr_542/a_7586/7586.pdf

Navarro, R. (2003). El rendimiento académico, concepto, investigación, desarrollo. Revista Iberoamericana sobre la Calidad, Eficacia, y Cambio de Educación.1 (2), 3. Recuperado de http://www.redalyc.org/pdf/551/55110208.pdf

Navarro, R. (2003). El rendimiento académico: concepto, investigación y desarrollo. Revista Electrónica Iberoamericana sobre Calidad, Eficacia y Cambio en Educación, 1(2), 1-15. Recuperado de https://revistas.uam.es/index.php/reice/article/viewFile/5354/5793

OMS (2016) Eliminación de las tareas escolares. Recuperado de: https://www.espaciologopedico.com/noticias/det/7251/la-onu-pide-prohibir-las-tareas-escolares.html

Pan, I., Regueiro, B., Ponte, B., Rodríguez., S., Piñeiro, I., y Valle, A. (2013). Motivación, implicación en los deberes escolares y rendimiento académico. Aula abierta, 41(3), 13-22. Recuperado de: https://dialnet.unirioja.es/servlet/articulo?codigo=4401103. Recuperado de: https://dialnet.unirioja.es/servlet/articulo?codigo=4401103 
Marjorie Narcisa Cevallos Rosero, Tania Estefanía Aguirre Espinosa, Edgar Efraín Obaco Soto...

Plan Nacional de Desarrollo. (2017). Senplades. Recuperado de: http://www.planificacion.gob.ec/wpcontent/uploads/downloads/2017/10/PNBV-26-OCT-FINAL_0K.compressed1.pdf

Plan decenal de educación del Ecuador (2006-2015). Ministerio de educación. Recuperado de: https://educacion.gob.ec/wp-content/uploads/downloads/2012/08/Rendicion_2007.pdf

Posada, M., Taborda, A. (2012). Reflexiones sobre la pertinencia de las tareas escolares: acercamientos para futuros estudios. Uni-pluri/versidad, 12(2), $22 \quad-33 . \quad$ Recuperado de: https://aprendeenlinea.udea.edu.co/revistas/index.php/unip/article/viewFile/14433/12669

Quispe., W. (2017). La funcionalidad de la tarea. Revista para el aula, (21). 36-37. Recuperado de: https://www.usfq.edu.ec/publicaciones/para_el_aula/Documents/para_el_aula_21/pea_021_0018.pdf

Real Academia Española (Ed). (2017). Diccionario de la lengua española. Recuperado de: http://dle.rae.es/?id=ZC79NI2

Regueiro, B., Suárez, N., Valle, A., Núñez, J., y Rosário, P. (2015). La motivación e implicación en los deberes escolares a lo largo de la escolaridad obligatoria. Revista de Psicodidáctica, 20 (1), 47-63. Doi: 10.1387/RevPsicodidact.12641

Rodríguez, J. (2009). Plataformas De Enseñanza Virtual Para Entornos Educativos. Pixel-Bit. Revista de Medios y Educación, (34), 217-233. Recuperado de: http://www.redalyc.org/pdf/368/36812036015.pdf

Rosario, G. (2018). El uso del aprendizaje basado en problemas en la enseñanza universitaria. Análisis de las competencias adquiridas y su impacto. Revista mexicana de investigación educativa, 23(76). 73-93. Recuperado de: http://www.scielo.org.mx/pdf/rmie/v23n76/1405-6666-rmie-23-76-73.pdf

Rosario, P., Mourão, R., Baldaque, M., Nunes, T., Nuñez, J., Gonzalez-Pienda, J., Cerezo, R., y Valle, A. (2009). Tareas para casa, autorregulación del aprendizaje y rendimiento en matemáticas. Revista de Psicodidáctica, 14 (2), 179-192. Recuperado de: https://www.redalyc.org/revista.oa?id=175

Rosario, P., Mourao, R., Trigo, L., Suarez, N., Fernández, E., Tuero-Herrero, E. (2011). Uso de diarios de tareas para casa en el inglés como lengua extranjera: evaluación de pros y contras en el aprendizaje autorregulado y rendimiento. Psicothema, 23 (4), 681-687. Recuperado de: http://www.psicothema.com/psicothema.asp?id=3941

Rosario, P., Mourao, R., Blandaque, M., Nunes, T., Núñez, J., Gonzales-Pineida, J., Cerezo, R., Valle, A. (2009). Tareas para casa, autorregulación del aprendizaje y rendimiento en matemáticas. Revista de Psicodidáctica, 14 (2), 179-192. Recuperado de: http://www.redalyc.org/articulo.oa?id=17512724002

Suárez, N., \& Núñez, J., \& Vallejo, G., \& Cerezo, R., \& Regueiro, B., \& Rosario, P. (2014). Tareas para casa, rendimiento académico e implicación de padres y profesores. International Journal of Developmental and Educational Psychology, 7 (1), 417-423. Recuperado de: https://www.redalyc.org/pdf/3498/349851791041.pdf

Suárez, N., Estrella, F., Cerezo, R., Rodríguez, C., Rosário, P., y Núñez, J. (2012). Tareas para casa, implicación familiar y rendimiento académico. Aula Abierta 40, (1). 73-84. Recuperado de: http://digibuo.uniovi.es/dspace/bitstream/10651/17589/1/AulaAbierta.2012.40.1.73-84.pdf

Tamayo, M., Tamayo. (2004). El proceso de investigación científica. México D. F.: Limusa.

Travé, G.; Estepa, J. y Delval, J. (2017). Análisis de la fundamentación didáctica de los libros de texto de conocimiento del medio social y cultural. Educación XX1, 20(1), 319-338, doi: 10.5944/ educXX1.11831

Valle, A., Pan, I., Núñez, J., Rosário, P., Rodríguez, S., y Regueiro, B. (2015). Deberes escolares y rendimiento académico en Educación Primaria. Anales de Psicología, 31 (2), 562-569. Recuperado de: http://www.redalyc.org/articulo.oa?id=16738685019

Vázquez, C. (2016). Factores Asociados al Fracaso Escolar en la Educación Secundaria de Huelva. REICE. Revista Iberoamericana sobre Calidad, Eficacia y Cambio en Educación, 14 (3), 131-144. Doi: 10.15366/reice2016.14.3.007

Velásquez, R. (2011). Dosificación De Tareas Escolares Y Aprendizaje Significativo Del Niño Del CEC (Tesis de grado) Universidad Rafael Landívar, Guatemala. Recuperado de: http://biblio2.url.edu.gt/Tesis/05/08/VelasquezRodolfo/Velasquez-Rodolfo.pdf 